\title{
Estado nutricional, consumo alimentar e satisfação corporal de gestantes do interior do Ceará
}

\section{Nutritional status, food consumption and body satisfaction of pregnant women in the interior of Ceará}

Jane Kelly Rodrigues Girão, Fernanda Thais de Oliveira Lima²

1. ORCID: https://orcid.org/0000-0002-2513-7084. Acadêmica. Instituto Federal de Educação, Ciência e Tecnologia do Ceará - IFCE, Rua Estevão Remígio de Freitas, Monsenhor Otávio, 62930-000, Limoeiro do Norte, Ceará, Brasil. E-mail: janek.girao@ gmail.com

2. ORCID: https://orcid.org/0000-0002-3459-4738. Nutricionista. Instituto Federal de Educação, Ciência e Tecnologia do Ceará - IFCE, Rua Estevão Remígio de Freitas, Monsenhor Otávio, 62930-000, Limoeiro do Norte, Ceará, Brasil. E-mail: fernandanutri14@ gmail.com.

CONTATO: Autor correspondente: Jane Kelly Rodrigues Girão | Endereço: Estevão Remígio de Freitas,998, Centro.Telefone: 88-988575480.E-mail: janek.girao@gmail.com

RESUMO O objetivo do trabalho foi analisar o estado nutricional, consumo alimentar e satisfação corporal de gestantes atendidas em duas Unidades Básicas de Saúde (UBS) em Limoeiro do Norte/CE. Foi realizado o cálculo do IMC gestacional e pré-gestacional e aplicado dois recordatórios alimentares de 24h, com o qual calculou-se a adequação de micronutrientes e valor calórico. Foi utilizado Body Shape Questionnaire (BSQ) para avaliar a satisfação corporal. No período pré-gestacional prevaleceu o excesso de peso para $60 \%$ das mulheres e $67,5 \%$ durante a gestação. Quanto aos micronutrientes todas as gestantes estavam com o consumo possivelmente abaixo do recomendado e apenas $7,5 \%$ apresentaram consumo energético possivelmente adequado. 
Foi constatado uma correlação negativa e significativa $(p=0,622 ; r=-0,788)$ entre o IMC e BSQ. Houve uma elevada prevalência de excesso de peso nas gestantes, bem como um consumo alimentar possivelmente inadequado de micronutrientes e energético. As gestantes com maiores IMC apresentaram menor satisfação corporal.

DESCRITORES: Gestantes. Consumo de alimentos. Imagem Corporal. Estado Nutricional

ABSTRACT The objective of this study was to analyze the nutritional status, food consumption and body satisfaction of pregnant women attended at two Primary Health Units (UBS) in Limoeiro do Norte/CE. The gestational and pre-gestational BMI was calculated and two 24-hour food records were applied, with which the adequacy of micronutrients and caloric value were calculated. The Body Shape Questionnaire (BSQ) was used to assess body satisfaction. In the pre-pregnancy period, overweight prevailed for $60 \%$ of women and $67.5 \%$ during pregnancy. As for micronutrients, all pregnant women had consumption possibly below the recommended level and only $7.5 \%$ had possibly adequate energy consumption. A negative and significant correlation $(p=0.622 ; r=-0.788)$ was found between BMI and BSQ. There was a high prevalence of overweight among pregnant women, as well as possibly inadequate food intake of micronutrients and energy. Pregnant women with higher BMI had lower body satisfaction.

DESCRIPTORS: Pregnant women. Food consumption. Body image. Nutritional status 


\section{INTRODUÇÃO}

O. período gestacional configura-se por intensas alterações fisiológicas, endócrinas e metabólicas, que levam a modificações nas necessidades nutricionais e na ingestão alimentar da mãe. O ganho de peso e o estado nutricional durante a gestação influenciam na saúde do feto. Tanto o déficit de peso quanto o ganho de peso materno constituem fatores de risco para diversas doenças, como diabetes gestacional, hipertensão arterial e anemia ferropriva1.

Durante a gestação, a dieta deve ser constituída por alimentos variados, levando em consideração os hábitos alimentares individuais, com objetivo de atender as necessidades energéticas e nutricionais fundamentais para o período de vida da mulher. A importância sobre um adequado estado nutricional deve ser ressaltada para as mulheres gestantes, com objetivo de prevenir problemas na saúde na mãe como também no feto².

Um plano alimentar balanceado durante todos os períodos da gestação é um método eficaz para garantir o pleno crescimento e desenvolvimento do feto. A vitamina A possui influencia no período em que as células se proliferam e se diferenciam, como na gestação e na primeira infância. As vitaminas do complexo $B$, por sua vez, participam de diversos processos do organismo, como síntese de energia, oxirredução e síntese de DNA, também tem sido empregada no tratamento de náuseas e vômitos na gestação ${ }^{3}$.

As transformações que acontecem no decorrer da gestação trazem para a gestante uma série de dúvidas e questionamentos em relação ao estado físico e emocional, dentre estes destaca-se a satisfação ou insatisfação com a imagem corporal que sucede das semanas de gestação. O padrão de beleza imposto pela sociedade interfere na percepção de imagem corporal das mulheres, inclusive de gestantes, que podem optar por consumir dietas restritivas tornando este período uma fase de insatisfação corporal ${ }^{4}$.

A importância sobre um adequado estado nutricional deve ser ressaltada para as muIheres gestantes, com objetivo de prevenir problemas na saúde na mãe como também no feto. Durante a gravidez, as mulheres sofrem diversas mudanças no corpo e no peso, sendo esses fatores contribuintes de forma negativa ou positiva na satisfação corporal durante a gestação. Baixos níveis de satisfação corporal estão associados a ganho de peso excessivo, depressão e desordens alimentares, que refletem negativamente na saúde da mãe e do bebê5 .

A qualidade da alimentação tem um papel importante no estado nutricional de gestantes, se realizada de forma inadequada pode apresentar riscos desfavoráveis para mãe e para o concepto. Ademais, vivemos em uma cultura que admira a magreza e por isso o ganho de peso durante a gravidez pode se tornar um problema. Assim, é relevante estudar o consumo alimentar e satisfação corporal como fatores contribuintes no estado nu- 
tricional desse público específico, proporcionando dados referentes a educação nutricional para gestantes, assim, nas orientações de saúde em geral. Além disso, foi relevante para a elaboração do projeto a escassez das pesquisas desenvolvidas acerca do tema.

O presente estudo teve como objetivo analisar a satisfação corporal, consumo alimentar e estado nutricional de gestantes atendidas em duas unidades básicas de saúde em Limoeiro do Norte/CE.

\section{MÉTODO}

Trata-se de um estudo descritivo, transversal e de abordagem quantitativa realizado em duas Unidades Básicas de Saúde localizada no município de Limoeiro do Norte Ceará. A princípio a amostra era de 80 gestantes, porém quando se iniciou a coleta de dados apenas 71 gestantes estavam sendo acompanhadas pela equipe da UBS. Após exclusão de 13 mulheres com doenças crônica e menores de idade, e 17 gestante que não compareceram para a consulta durante o período de coleta de dados, a amostra contou com 40 participantes do estudo.

Primeiramente, foi lido e explicado o Termo de Consentimento Livre e Esclarecido (TCLE) para cada gestante, que decidiram por fazer parte do estudo ou não. A coleta iniciou com aplicação um questionário sobre dados pessoais (nome, idade, estado civil e grau de escolaridade) e dados gestacionais (semana gestacional, peso pré-gestacional e intercorrências em gestação anterior). Logo após, foi coletado o peso e a estatura da gestante, para realização do cálculo do Índice de Massa Corporal (IMC) atual, realizado através da divisão do peso pela altura ao quadrado, onde a partir do resultado, as gestantes foram classificadas quanto ao estado nutricional através da tabela de IMC por idade gestacional ${ }^{6}$. Também foi verificado o IMC pré-gestacional, através da divisão do peso pré-gestacional referido pela gestante pela estatura ao quadrado ${ }^{7}$. Os pesos foram aferidos em balança digital da marca Toledo Prix ${ }^{\circledR}$ com capacidade de $200 \mathrm{~kg}$ e precisão de $50 \mathrm{~g}$. As estaturas foram mensuradas em estadiômetro acoplado à balança com precisão de $1,0 \mathrm{~mm}$, com as participantes em pé, em posição firme, com os braços relaxados e cabeça no plano horizontal.

Para avaliação do consumo alimentar foi aplicado dois recordatórios alimentares de 24 horas (R24h). Utilizou-se formulário próprio e um álbum fotográfico das porções alimentares para maior precisão da coleta de informações. Não foi incluído no estudo o consumo de suplementos alimentares pelas gestantes. Os dados do R24h, em medidas caseiras, foram convertidos em gramatura através da tabela de medidas caseiras ${ }^{8}$ e a composição em termos calóricos e de micronutrientes foi determinada com a Tabela de Composição Química dos Alimentos (TACO). 
Para análise de adequações do consumo de micronutrientes, foram considerados os valores das Dietary Reference Intakes (DRI), sendo a amostra separada em dois grupos, gestantes de 19 a 30 anos e 31 a 50 anos, pois possuem referências diferentes para micronutrientes. Foram avaliados ferro, folato, vitamina $A$ e vitamina B6, baseando-se em suas respectivas necessidades médias estimada $(E A R)^{9-10}$. $O$ valor calórico encontrado foi confrontado com a necessidade energética estimada (EER) para gestantes eutróficas, e gasto total de energia (TEE) para aquelas que apresentaram sobrepeso ou obesidade ${ }^{7}$.

A avaliação da satisfação corporal das gestantes, foi realizada através da utilização do questionário validado e autoaplicável Body Shape Questionnaire (BSQ) que é composto por 34 questões em uma escala Likert, as gestantes foram classificadas de acordo com os resultados: pontuação abaixo de 80 ausência de distorção; pontuação entre 80 a 110 distorção leve; pontuação entre 110 e 140, distorção moderada; e classificação igual ou superior a 140 distorção grave ${ }^{11}$.

As variáveis quantitativas foram apresentadas em estatística descritiva como média, frequência simples e percentual. Foi aplicado a correlação de Pearson para verificar a relação do estado nutricional atual das gestantes e a satisfação corporal, utilizando o programa SPSS Statistics versão 20.0.

O estudo foi aprovado pelo Comitê de Ética em Pesquisa do Instituto Federal de Educação, Ciência e Tecnologia do Ceará, atendendo a resolução n 466/2012, sob parecer número 3.505.429.

\section{RESULTADOS}

De acordo com avaliação nutricional, no período pré-gestacional prevaleceu o excesso de peso para $60 \%$ das mulheres ( $45 \%$ sobrepeso e $15 \%$ obesidade). Durante o acompanhamento gestacional esse percentual aumentou para $67,5 \%$ das mulheres ( $45 \%$ sobrepeso e $22,5 \%$ obesidade). (Gráfico 1 ) 
Gráfico 1. Estado nutricional pré-gestacional e gestacional de gestantes atendidas em duas unidades básicas de saúde em Limoeiro do Norte, Ceará,2019.

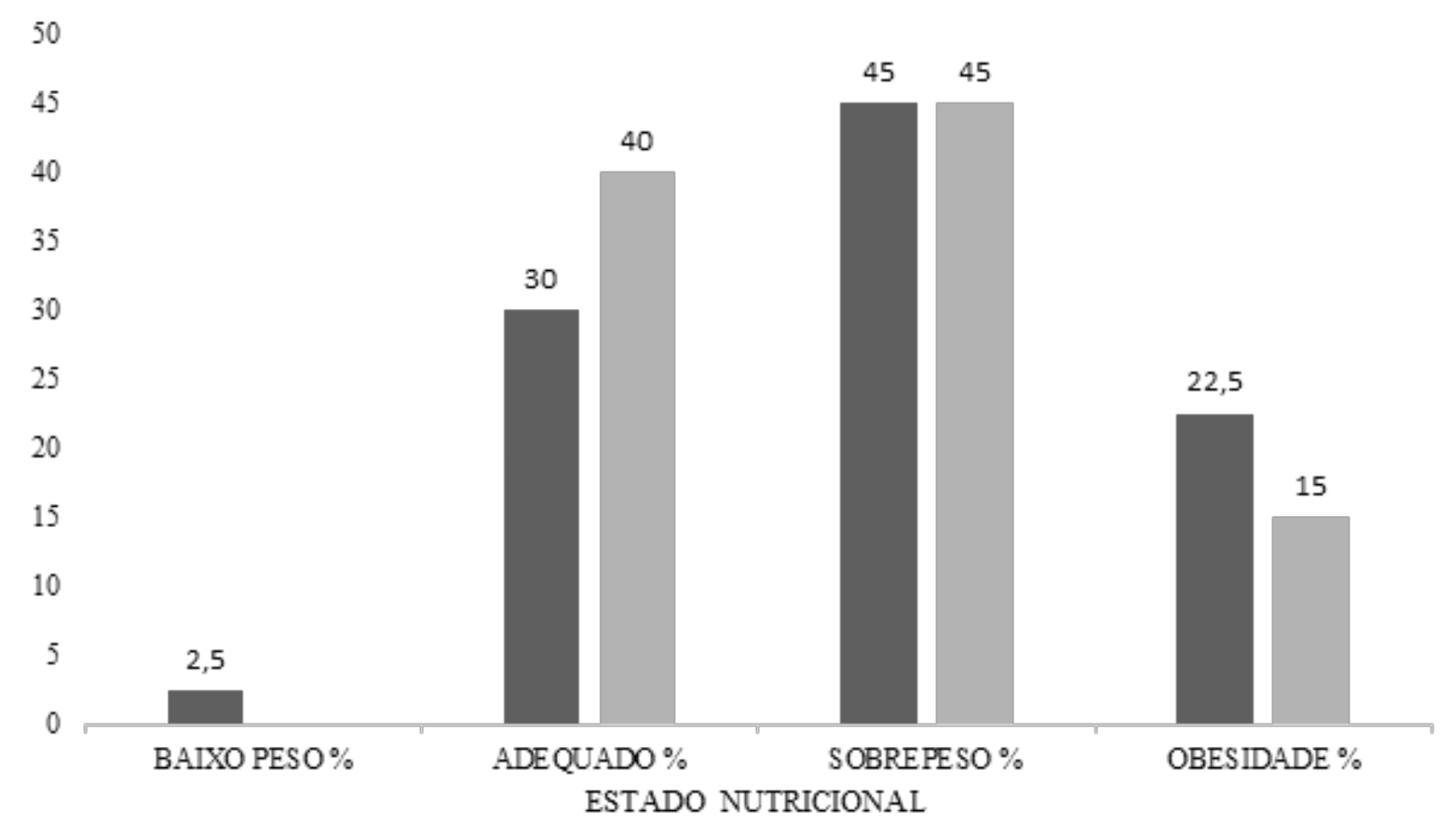

- IMC GESTACIONAL $\quad$ IMC PRÉ-GESTACIONAL

Fonte: Elaborada pelos autores, 2020. Legenda: IMC: índice de massa corporal

A análise de adequação dos micronutrientes mostrou que todas as gestantes de ambas as faixas etárias estavam com o consumo abaixo do recomendado de vitamina B6/ piridoxina $(36,8 \% ; 25,6 \%)$, vitamina B9/folato $(2,9 \% ; 1,8 \%)$ e ferro $(32 \% ; 25,3 \%)$, estes com percentual de adequação inferior a $50 \%$, ou seja, o consumo qualitativo dessas gestantes possivelmente está inadequado para esses micronutrientes. (Tabela 1)

Tabela 1. Consumo de micronutrientes das gestantes atendidas em duas unidades básicas de saúde em Limoeiro do Norte, Ceará,2019.

\begin{tabular}{|c|c|c|c|c|c|}
\hline NUTRIENTE & $\begin{array}{c}\text { EAR } \\
\text { (19-50 anos) }\end{array}$ & $\begin{array}{c}\text { Média de } \\
\text { consumo } \\
\text { (19-30 } \\
\text { anos) }\end{array}$ & $\begin{array}{c}\text { Média de } \\
\text { consumo } \\
(31-50 \\
\text { anos) }\end{array}$ & $\begin{array}{c}\% \\
\text { Adequação } \\
\text { (19 -30 anos) }\end{array}$ & $\begin{array}{c}\% \\
\text { Adequação } \\
\text { (31-50 anos) }\end{array}$ \\
\hline Vitamina B6 (mg) & 1,6 & 0,59 & 0,41 & 36,8 & 25,6 \\
\hline Folato $(\mu \mathrm{g})$ & 520 & 15,16 & 9,36 & 2,9 & 1,8 \\
\hline Vitamina A $(\mu \mathrm{g})$ & 550 & 225,10 & 275,21 & 40,9 & 50,5 \\
\hline Ferro (mg) & 22 & 7,04 & 5,58 & 32 & 25,3 \\
\hline
\end{tabular}

Fonte: Elaborada pelos autores, 2020. Legenda: EAR: necessidade média estimada; mg: miligrama; $\mu \mathrm{g}$ : micrograma

Apenas $7,5 \%$ das gestantes que frequentam as unidades básicas de saúde apresentavam consumo energético possivelmente adequado, ou seja, quando se fala em 
consumo alimentar quantitativo pode-se dizer que está inadequado para as gestantes avaliadas. (Tabela 2).

Tabela 2. Avaliação do consumo energético das gestantes atendidas em duas unidades básicas de saúde em Limoeiro do Norte, Ceará,2019.

\begin{tabular}{llll}
\hline Consumo energético do grupo(kcal) & $\mathrm{N}$ & $\%$ & Média \\
\hline POS INA & 37 & 92,5 & $1.467,57$ \\
POS ADE & 3 & 7,5 & $2.153,06$ \\
\hline
\end{tabular}

Fonte: Elaborada pelos autores, 2020. Legenda: POS INA = possivelmente inadequado; POS ADE = possivelmente adequado.

Relacionou-se o estado nutricional com a classificação das gestantes no $B S Q$, foi constatado uma correlação negativa e significativa $(p=0,622 ; r=-0,788)$ entre esses dois parâmetros, ou seja, à medida que aumentava o número de gestantes com maiores IMC o percentual de gestantes com maior pontuação no BSQ diminuía. (Gráfico 2)

Gráfico 2. Frequência de gestantes que apresentaram satisfação ouinsatisfação com a imagem corporal conforme o estado nutricional atual em Limoeiro do Norte, Ceará, 2019.

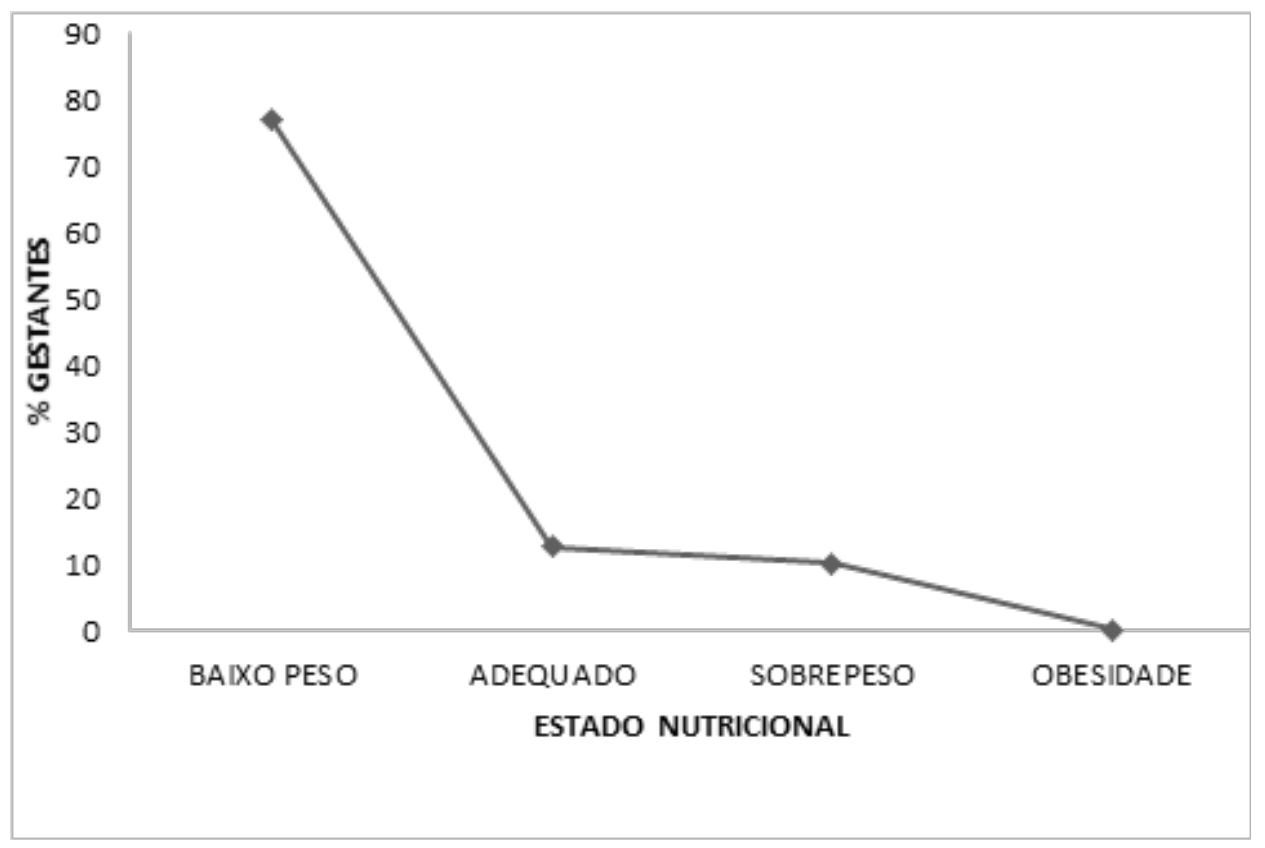

Fonte: Elaborada pelos autores, 2020.

\section{DISCUSSÃO}

O estado nutricional pré-gestacional é uma das condições associadas ao ganho de peso durante a gravidez. Um resultado importante encontrado na amostra estudada foi que $60 \%$ das mulheres apresentaram excesso de peso pré-gestacional e $67,5 \%$ durante a gestação. É importante ressaltar que nesse estudo, as mulheres com IMC inadequado 
não apresentavam problemas ou distúrbios relacionados a gravidez, diabetes gestacional, hipertensão, anemia ferropriva e dentre outra. Um estudo realizado no município de Colombo, no Paraná, houve uma prevalência de excesso de peso, com 40,8\% das participantes nesse estado nutricional no período pré-gestacional e durante a gestação $(46,2 \%)^{12}$.

É relevante enfatizar, para essas mulheres, a importância do ganho de peso adequado durante a gestação, pois previne problemas de saúde tanto na mãe quanto no feto $^{13}$. Quanto ao período gestacional, o ganho de peso inadequado é um risco para as gestantes obesas, que estão passíveis a desenvolver diabetes gestacional, hipertensão arterial que podem ocasionar complicações no trabalho de parto, além da criança ficar mais predisposta ao risco de mortalidade perinatal e má formação fetal ${ }^{14}$.

Com relação ao consumo de micronutrientes, o baixo percentual de adequação do consumo de vitamina B6 (piridoxina) encontrado nesse estudo, corrobora com um trabaIho que analisou o nível plasmático de vitamina B6, mostrando que cerca de $35,4 \%$ das gestantes avaliadas apresentaram deficiência nessa vitamina e quase todas as mulheres $(93,6 \%)$ engravidaram com pelo menos uma deficiência de micronutriente ${ }^{15}$. Baixos níveis de vitamina B6 podem levar ao aumento da homocisteína com resultados adversos, incluindo descolamento de placenta, baixo peso ao nascer e parto prematuro ${ }^{16}$.

O baixo percentual de adequação do consumo de ácido fólico (folato) pelas gestantes é preocupante, pois a deficiência de folato resulta no acúmulo de homocisteína, que pode aumentar o risco de resultados adversos, incluindo pré-eclâmpsia e anomalias fetais $^{17}$. Um estudo controlado randomizado realizado na California, mostrou que 40 a $50 \%$ das participantes tinham uma ingestão inadequada de folato quando comparado os valores com as EARs ${ }^{18}$.

É importante ressaltar que não foi avaliado o consumo de suplementos pelas gestantes, e sabe-se que a suplementação de folato durante a gravidez é recomendado. Além disso, no Brasil há uma legislação para fortificação de farinhas com ferro e ácido fólico, o que poderia não está comtemplado na tabela de composição de alimentos que foi utilizada para quantificar os nutrientes consumidos.

O consumo de vitamina A (retinol) pelo grupo estudado mostrou que 50,5\% das muIheres estavam com o um percentual de adequação no consumo adequado. Um estudo realizado em três regiões do nordeste do Brasil mostrou que na amostra de 676 gestantes, a prevalência cumulativa de vitamina A deficiente ou baixa foi de $6,2 \%$, com a grande maioria das mulheres com níveis aceitáveis de retinol sérico ${ }^{19}$. Esse consumo possivelmente adequado pode ser justificado devido a facilidade dessa vitamina ser encontrada em diversos alimentos, como carnes, hortaliças, legumes e frutas ${ }^{20}$. Apesar do consumo adequado de vitamina A por metade das gestantes do estudo, e da grande diversidade de frutas e hortaliças ricas nessa vitamina na região nordeste, a hipovitaminose A consti- 
tui um problema de saúde pública nessa região, e em outras do Brasil, sendo identificada como a mais grave da América Latina ${ }^{21}$.

Em relação ao mineral ferro, os percentuais de adequação de consumo em ambas as faixas etárias das gestantes foram inferiores a $50 \%$. Outro estudo destacou que na Europa 24,5\% das mulheres grávidas entre 15 e 49 anos têm anemia causada, principalmente, por deficiência de ferro ${ }^{22}$. Na gravidez, a demanda por ferro aumenta para atender a necessidades fetais e as perdas. A deficiência de ferro tem sido associada a maior risco de nascimento prematuro, função materna comprometida e defesas contra infecções diminuídas, bem como desenvolvimento psicomotor e função cognitiva anormal na infância ${ }^{17}$.

Além disso, estudos mostraram que a deficiência de vitamina A pode afetar o transporte de ferro e a produção de eritrócitos, além de prejudicar a mobilização de ferro das reservas. Por isso, muitos pesquisadores recomendam que a suplementação de ferro deve ser acompanhada por medidas de controle também em relação a vitamina $A^{21}$.

O baixo consumo desses nutrientes pelo grupo estudado é um reflexo do padrão alimentar das gestantes. Um estudo transversal observou que as gestantes avaliadas apresentaram baixo consumo diário de leite e derivados, de ovos e das carnes e alto consumo de refrigerantes, doces e sorvetes. Portanto, considera-se que uma alimentação nutricionalmente inadequada durante a gestação é um fator que compromete o aporte energético e de nutrientes, levando a repercussões maternas e fetais ${ }^{23}$.

Neste trabalho, avaliou-se o percentual de adequação do consumo de ferro apenas pelos dados dietéticos, não sendo considerado a suplementação. No Brasil, poucos são os estudos recentes sobre deficiência de micronutrientes em gestantes, principalmente através de avaliação dietética.

Quanto ao aporte calórico, apenas três gestantes estavam com o consumo possivelmente adequado. Em uma pesquisa realizada com gestantes atendidas em Unidades Básicas de Saúde (UBS) do município de Maceió, mostrou que a média do consumo energético encontrado foi abaixo do padrão de referência. $O$ baixo consumo energético da maioria das gestantes pode ser reflexo de uma dieta inadequada e com pouca variedade de alimentos que não fornece suprimento adequado de energia para atender aos requisitos habituais da mãe bem como os do feto em crescimento. Além disso, durante a gravidez a demanda de energia é maior para a síntese de novos e crescimento de tecidos existentes ${ }^{23}$.

Cabe ressaltar, que a maioria das gestantes apresentaram um baixo consumo energético, entretanto a frequência de excesso de peso foi elevada. Assim, é importante destacar que o Recordatório de $24 \mathrm{~h}$ requer da memória dos participantes, e que muitos não conseguem lembrar detalhadamente do que foi consumido, além de que, a obesidade 
tem etiologia multifatorial.

A satisfação corporal apresentou correlação negativa com o IMC, ou seja, as gestantes com maiores IMC apresentaram um nível maior de distorção da imagem corporal, indicando menor satisfação corporal. O mesmo resultado se mostrou presente em um estudo realizado em Minas Gerais, as gestantes com sobrepeso e obesidade apresentaram maior insatisfação corporal quando comparadas às de baixo peso. Durante o terceiro trimestre, as gestantes tendem a ter atitude mais negativas relacionadas ao corpo ${ }^{13}$. Deste modo, uma avaliação da satisfação corporal durante a gravidez pode auxiliar os profissionais da saúde identificar as mulheres que estão vulneráveis ao ganho de peso inadequado e que podem aderir a dietas restritivas. Além disso, destaca-se a importância de avaliar os fatores psicológicos em gestantes, devido as implicações que podem refletir na saúde da mãe e do bebê.

Dentre as limitações desse estudo, destaca-se a utilização do R24h alimentar no qual as informações foram referidas pelas mulheres, o que pode levar a erros por depender da memória, o mesmo se aplica ao peso pré-gestacional, que pode ter sido um valor super ou subestimado. Outro problema inerente ao estudo foi o tamanho e perda da população, porém vale ressaltar a dificuldade no recrutamento das gestantes por muitas não comparecerem para as consultas. Por fim, a inexistência de instrumentos criados e validados no Brasil para avaliar a satisfação corporal desse público específico.

\section{CONCLUSÃO}

Conclui-se que houve uma prevalência de excesso de peso nas gestantes atendidas nas Unidade Básicas de Saúde, bem como um consumo de micronutrientes (vitamina B6/piridoxina, ácido fólico/folato e ferro) e energético possivelmente inadequado. Além disso, as gestantes com maiores IMC apresentaram menor satisfação corporal. Diante do contexto analisado, sugere-se a introdução de ações de orientação nutricional durante o pré-natal e assistência pré-natal pautada numa abordagem interdisciplinar, com a participação de nutricionista, psicólogo, educador físico, dentre outros, para minimizar as distorções de imagem corporal, empoderar as gestantes, garantir uma boa nutrição e bem-estar durante o período gestacional.

\section{AGRADECIMENTOS E CONFLITOS DE INTERESSE}

A Secretaria Municipal de Saúde do Município de Limoeiro do Norte, pelo recrutamento das participantes do estudo. O presente estudo não possui conflitos de interesse entre os autores.

\section{REFERÊNCIAS}


1.Rosa RL, Molz P, Pereira CS. Perfil nutricional de gestantes atendidas em uma unidade básica de saúde. Cinergis. 2014 [acesso em 2020 mai 14];15(2):98-102. Disponível em: https://online.unisc.br/seer/index. php/cinergis/article/view/5134

2.Gomes CB, Vasconcelos LG, Cintra RMGC, Dias LCG, Carvalhaes MABL. Hábitos alimentares das gestantes brasileiras: revisão integrativa da literatura. Ciência e Saúde Coletiva. 2019 [acesso em 2020 mai 18];24(6): 2293- 2306. https://doi.org/10.1590/1413-81232018246.14702017

3.Almeida CAN, Pimentel C, Fonseca EB. Além da nutrição: o impacto da nutrição materna na saúde das futuras gerações. Copyrigh. $1^{\circ}$ edição. São Paulo. 2019.

4. Teixeira FA, Schneider VLP, Wolpe RE, Sperandio FF. Satisfação com a imagem corporal em mulheres gestantes e não gestantes. ABCS Health Sciences.2015 [acesso em 2020 mai 21]; 40(2):69-74. Disponível em: http://dx.doi.org/10.7322/abcshs.v40i2.733

5.Pullmer R, Zaitsoff S, Cobb R. Body Satisfaction During Pregnancy: The Role of Health-Related Habit Strength. Maternal and Child Health Journal. 2018 [acesso em 2020 mai 24]]; (22):391-400. Disponível em: https://doi.org/10.1007/s10995-017-2406-9

6.Atalah SE, Castillo LC, Castro SR, Amparo AP. Propuesta de um nuevo estándar de Evaluación nutricional em embarazadas. Revista Medicina Chile. 1997 [acesso 2020 mai 27];125(12):1429-1436.Disponível em: https://link.springer.com/article/10.1007/s10995-017-2406-9

7.Vitolo MR. Nutrição da gestação ao envelhecimento. Rio de Janeiro: Rubio;2015.

8.Pinheiro ABV, Lacerda EMA, Costa VM, Benzecry EH, Gomes MCS. Tabela para avaliação de consumo alimentar em medidas caseiras. São Paulo: Atheneu; 2008.

9.Institute of Medicine, Food and Nutrition Board. Dietary Reference Intakes. Thiamin, Riboflavin, Niacin, Vitamin $\mathrm{B}_{6}$, Folate, Vitamin $\mathrm{B}_{12}$, Pantothenic Acid, Biotin, and Choline. Washington: National Academy Press; 1998.

10.Institute of Medicine, Food and Nutrition Board. Dietary Reference Intakes. Dietary Reference Intakes for Vitamin A, Vitamin K, Arsenic, Boron, Chromium, Copper, lodine, Iron, Manganese, Molybdenum, Nickel, Silicon, Vanadium, and Zinc. Washington: National Academy Press; 2001.

11.Freitas SR. Instrumentos para a avaliação dos transtornos alimentares: Transtornos alimentares e obesidade. Porto Alegre: Artmed; $1^{\circ}$ edição,2006.

12. Manera F, Hofelmann DA. Excesso de peso em gestantes acompanhadas em unidades de saúde de Colombo, Paraná, Brasil. Demetra. 2019 [acesso em 2020 mai 24]; 14:1-16. Disponível em: https://doi. org/10.12957/demetra.2019.36842

13. Meireles JFF, Neves CM, Carvalho PHB, Ferreira MEC. Satisfação corporal, idade gestacional e estado nutricional em gestantes. ABCS Health Sci. 2016 [acesso em 2020 mai 28; 41(1):23-28. Disponível em: http://dx.doi.org/10.7322/abcshs.v41i1.841

14.Teixeira CSS, Cabral ACC. Avaliação nutricional de gestantes sob acompanhamento em serviços de pré-natal distintos: a região metropolitana e o ambiente rural. Revista Brasileira Ginecologia Obstetrícia. 2016 [acesso em 2020 mai 29]; 38:27-34. Disponível em: http://dx.doi.org/10.1055/s-0035-1570111

15.Schulze KJ, Sucheta M, Saijuddin S, Hasmot A, Shamim AA, Wu LSF et al. Antenatal Multiple Micronutrient Supplementation Compared to Iron-Folic Acid Affects Micronutrient Status but Does Not Eliminate Deficiencies in a Randomized Controlled Tria: Among Pregnant Women of Rural Bangladesh. The Journal of Nut/rition.2019 [acesso em 2020 abr 02];149:1260-1270. Disponível em: https://doi.org/10.1093/jn/ nxz046

16. Rogne T, Tielemans M.J, Chong MFF, Yajnik CS, Krishnaveni GV, Poston L et al. Maternal vitamin B12 in pregnancy and risk of preterm birth and low birth weight: A systematic review and individual participant data meta-analysis. Am. J. Epidemiol. 2017 [acesso 2020 abr 04]; 185:212-223.Disponível em: https:/l www.ncbi.nlm.nih.gov/pmc/articles/PMC5390862/

17.Mousa A, Nagash A, Lim S. Macronutrient and Micronutrient Intake during Pregnancy: An Overview of Recent Evidence. Nutrients. 2019 [acesso em 2020 abr 04]; 11(443). Disponível em: https://doi.org/10.3390/ 
nu11020443

18.Phelan S, Abrams B, Wing RR. Prenatal Intervention with Partial Meal Replacement Improves Micronutrient Intake of Pregnant Women with Obesity. Nutrients. 2019; 11. Disponível em: https://doi.org/10.3390/ nu11051071

19.Maia SB, Caminha MFC, Silva SL, Souza ASR, Santos CC, Filho MB. The Prevalence of Vitamin A Deficiency and Associated Factors in Pregnant Women Receiving Prenatal Care at a Reference Maternity Hospital in Northeastern Brazil. Nutrients. 2018 [acesso 2020 abr 10];10 (1271). Disponível em: https://doi. org/10.3390/nu10091271

20.El Beitune P, Jimenez MF, Salcedo MMBP, et al. Nutrição durante a gravidez. São Paulo: Federação Brasileira das Associações de Ginecologia e Obstetrícia, $1^{\circ}$ edição,2018

21.Cozzolino SMF. Biodisponibilidade de nutrientes. São Paulo: Manole; $5^{\circ}$ edição,2016

22. Milman N, Taylor CL, Merke J, Brannon PM. Iron status in pregnant women and women of reproductive age in Europe. Am J Clin Nutr. 2017 [acesso em: 2020 abr 25]; 106. Disponível em: https://doi.org/10.3945/ ajcn. 117.156000

23.Ferreira RC. Bezerra AR. Tavares MCM. Micaely Tenório MCS, Barros AMR. Oliveira ACMO Padrão de consumo dietético de gestantes e sua relação com a insegurança alimentar no domicílio. BRASPEN J. 2017 [acesso em: 2020 abr 30]; 32 (2): 128-33. Disponível em: http://www.braspen.com.br/home/wp-content/uploads/2017/08/06-AO-Padrao-de-consumo-dietetico.pdf

RECEBIDO: 23/11/2020

ACEITO: 28/04/2021 